\section{Dr. Hersh replies}

\section{To the Editor:}

In their letter, Dr. Chanchlani, et al ${ }^{1}$ briefly described the outcomes of their established program for transition from pediatric to adult rheumatology care in the United Kingdom ${ }^{2,3}$. Their results demonstrated that even with a coordinated program, nearly one-quarter of the cohort did not meet their definition of a successful transfer (receipt of a letter from the first adult appointment), or was lost to followup. Further, the authors found that documentation of direct communication with adult providers was lacking in $24 \%$ of cases, and patients were not provided with a medical summary in nearly $60 \%$ of cases. As the authors discussed, written communication with adult providers and creation of a medical summary for patients have been included in the "Six Core Elements of Health Care Transition" document developed by the US Got Transition agency ${ }^{4}$. It is not clear from the results presented whether the lack of documented communication was associated with the success of the transfer process, but the authors raised this as a potential area of deficit in their existing transition program. They concluded that a team approach with coordination of care between pediatric and adult rheumatology services could improve transition outcomes.

I agree with the authors that communication between pediatric and adult rheumatology providers is essential to ensuring successful transfer of care. Ideally, the pediatric rheumatologist would provide a detailed summary of the patient's clinical and treatment course, as well as an assessment of their transition readiness and self-care abilities, and the provider of adult care would present a letter or some other documentation of the patient's successful transfer to adult care. Even better would be a verbal handoff by telephone or in person, in addition to written communication. Unfortunately, these are processes that can take a considerable amount of time, and may be difficult to achieve when trying to coordinate communication between busy or underresourced clinical practices, particularly if they do not use the same medical record system.

Given the potential barriers to successful communication between pediatric and adult care providers, it becomes essential that the patients feel comfortable advocating for themselves and their healthcare needs with their new provider. Ideally, the transitioning patient knows his or her health history, how to manage and anticipate health needs, and how to navigate what is often a new healthcare system. Although far from optimal, in the current model the healthcare system largely relies on patients and their families to bridge the gap between pediatric and adult care. Given this, many current transition programs promote the development of self-management and self-advocacy skills as an essential component of successful transition ${ }^{5,6}$.

As the authors of the letter demonstrated, lapses in care in the transition from pediatric to adult rheumatology were common, even with an established transition program in place. For patients with a chronic rheumatic disease, these lapses can have a significant effect on short-term and longterm health outcomes. Developing effective strategies to improve communication between providers may be a key component to reducing the transfer failure rate. Perhaps an ideal transition model is one in which, at a time that is appropriate based on patient disease control, developmental level, and transition readiness, transition-aged patients are (gently) "pushed" to adult care, while simultaneously the rheumatologists who treat adults are prepared to receive the patients and "pull" them in. Ideally, with the combination of a gentle "push" and a strong "pull," we can maintain continuity of care for young adults with rheumatic disease, thereby improving their health outcomes.

AIMEE O. HERSH, MD, MS, Assistant Professor, Department of Pediatrics, Division of Allergy, Immunology and Rheumatology, University of Utah,

Salt Lake City, Utah, USA. Supported by US National Institutes of Health, National Institute of Arthritis and Musculoskeletal and Skin Diseases (NIH NIAMS 1K23AR066064-01). Address correspondence to Dr. A.O. Hersh, 81 N. Mario Capecchi Drive, 4th Floor, Salt Lake City, Utah 84113, USA. E-mail: aimee.hersh@hsc.utah.edu

\section{REFERENCES}

1. Chanchlani N, McDonagh JE, McGee M. Informational continuity is integral for successful transition of adolescents. J Rheumatol 2015;42:901-2.

2. McDonagh JE, Shaw KL, Southwood TR. Growing up and moving on in rheumatology: development and preliminary evaluation of a transitional care programme for a multicentre cohort of adolescents with juvenile idiopathic arthritis. J Child Health Care 2006;10:22-42.

3. McDonagh JE, Southwood TR, Shaw KL; British Society of Paediatric and Adolescent Rheumatology. The impact of a coordinated transitional care programme on adolescents with juvenile idiopathic arthritis. Rheumatology 2007;46:161-8.

4. Got Transition. [Internet. Accessed February 4, 2015.] Available from: www.gottransition.org

5. Breakey VR, Ignas DM, Warias AV, White M, Blanchette VS, Stinson JN. A pilot randomized control trial to evaluate the feasibility of an Internet-based self-management and transitional care program for youth with haemophilia. Haemophilia 2014;20:784-93.

6. White M, Stinson JN, Lingley-Pottie P, McGrath PJ, Gill N, Vijenthira A. Exploring therapeutic alliance with an internet-based self-management program with brief telephone support for youth with arthritis: a pilot study. Telemed J E Health 2012;18:271-6.

J Rheumatol 2015;42:5; doi:10.3899/jrheum.150043 